This is a post-peer-review, pre-copyedit version of an article published in Advances in Computational Mathematics. The final authenticated version is available online at: https://doi.org/10.1007/s10444-014-9401-0

\title{
Numerical Reconstruction of Convex Polytopes from Directional Moments
}

\author{
Mathieu Collowald ${ }^{1}$, Annie Cuyt ${ }^{2}$, Evelyne Hubert ${ }^{1}$, Wen-shin Lee $^{2}$, and \\ Oliver Salazar Celis ${ }^{2}$ \\ ${ }^{1}$ GALAAD, INRIA Méditerranée, Sophia Antipolis, France \\ ${ }^{2}$ Department of Mathematics and Computer Science, University of Antwerp, Belgium
}

May 9, 2014

\begin{abstract}
We reconstruct an $n$-dimensional convex polytope from the knowledge of its directional moments up to a certain order. The directional moments are related to the projection of the polytope vertices on a particular direction. To extract the vertex coordinates from the moment information we combine established numerical algorithms such as generalized eigenvalue computation and linear interval interpolation. Numerical illustrations are given for the reconstruction of $2-\mathrm{d}$ and $3-\mathrm{d}$ convex polytopes.
\end{abstract}

\section{Introduction}

The reconstruction of the boundary of a shape from its moments is a problem that has only partially been solved. For instance, when the shape is a polygon [24, 13], or when it defines a quadrature domain in the complex plane [17], it has been proved that its boundary can be reconstructed exactly from the knowledge of its moments. Both results admit no obvious extension to higher dimensions. The technique in [6] is applicable in higher dimensions, but the authors reconstruct a shape's characteristic function, or more generally, a square-integrable function defined on a compact domain. The reconstruction algorithm for polygons is based on Davis' exact integration formula [7] of a function in the complex plane. For polynomial functions, Davis' formula can be seen as a low dimensional case of identities attributed to Brion [2]. Based on the latter, Gravin, Lasserre, Pasechnik and Robins proposed the reconstruction of an $n$-dimensional convex polytope in exact arithmetic [16].

Brion's integration formula over a polytope does not relate moment information directly to the vertices of the convex polytope, but rather to the projections of these vertices onto some 1-dimensional subspace. To recover the projections, we recognize an inverse problem that arises in several areas $[12,18,20,21,25]$ and can be solved numerically as a generalized eigenvalue problem.

After recovering the projections of the vertices on various one-dimensional subspaces, remains the problem of matching different projections (in different directions) of the same vertex, with 
that vertex. In this paper we describe how to solve this issue, without resorting to exact arithmetic. The problem cannot be solved with ordinary interpolation or least squares approximation. But using an interval interpolation technique [27], we understand why we need $n+1$ projections (or more) to solve the matching.

Our method is the result of combining techniques from quite different mathematical disciplines: integer lattices, computer algebra, numerical linear algebra, interval methods, inverse problems. The complete algorithm, which we demonstrate in Section 6 and challenge in Section 7, consists of the following steps:

1. The exact number of vertices $r$ is computed from an upper bound $R$ and moments up to order $2 R+1-n$, in a sample of directions.

2. For $n+1$ (or more) directions, the projections of the vertices are obtained as the generalized eigenvalues of a structured pair of matrices whose entries are determined from the directional moments up to order $2 r-n-1$.

3. Each of these projections is then matched to the corresponding vertex and its coordinates are computed as the coefficients of an $n$-dimensional interval interpolant.

The different steps in our algorithm involve Hankel matrices, in the singular value decomposition for the computation of $r$, as well as in the generalized eigenvalue problem delivering the vertex projections. Structured matrices with real elements have condition numbers that grow exponentially with their size [3], and the size of our matrices is determined by the number of vertices of the polytope. In Section 7 we are therefore required to use high precision floating-point arithmetic for the polyhedron with many vertices that represents a brilliant diamond cut.

The paper is organized as follows. In Section 2 we introduce geometric, complex and directional moments together with Davis' and Brion's formulae. In Section 3 we review Prony's method and the related eigenvalue problem to determine the projections of the vertices from the directional moments. In Section 4 we discuss the determination of the number of vertices. In Section 5 we present an algorithm to solve the matching problem. Numerical illustrations are given in the Sections 6 and 7 where we reconstruct 2 -d and 3-d convex polytopes.

\section{Geometric and directional moments}

In this section we present identities attributed to Brion. These identities are central in [1] to establish the complexity of the computation of the moments of a polytope. Brion's identities are also at the core of the solution of the inverse problem proposed in [16]. They can actually be seen as a generalisation of Davis' integration formula that was used to solve the shape-from-moment problem in 2-d [24, 13].

We consider a convex polytope in $\mathbb{R}^{n}$ determined by the set of its $r$ vertices $\mathcal{V}$. Abusing the notation, $\mathcal{V}$ also denotes the polytope itself.

The geometric moments are

$$
m_{\alpha}=\int_{\mathcal{V}} x^{\alpha} d x=\int \ldots \int_{\mathcal{V}} x_{1}^{\alpha_{1}} \ldots x_{n}^{\alpha_{n}} d x_{1} \ldots d x_{n}, \quad \alpha=\left(\alpha_{1}, \ldots, \alpha_{n}\right) \in \mathbb{N}^{n} .
$$


The order of the geometric moment $m_{\alpha}$ is $|\alpha|=\alpha_{1}+\ldots+\alpha_{n}$. These moments can be expressed as a multivariate polynomial in the coordinates of the vertices [28]. A complexity analysis for the computation based on Brion's identities is offered in [1] for exact arithmetic.

The moment in the direction $\delta \in \mathbb{R}^{n}$ of order $k$ is

$$
m_{k}(\delta)=\int_{\mathcal{V}}\langle x, \delta\rangle^{k} d x, \quad k \in \mathbb{N},
$$

where $\langle\cdot, \cdot\rangle$ denotes the usual scalar product in $\mathbb{R}^{n}$.

One can obtain any directional moment of order $k$ from the geometric moments of order $k$ with the multinomial formula

$$
m_{k}(\delta)=\sum_{|\alpha|=k}\left(\begin{array}{l}
k \\
\alpha
\end{array}\right) m_{\alpha} \delta^{\alpha} .
$$

Conversely, geometric moments of order $k$ can be obtained from the directional moments of order $k$ in $\left(\begin{array}{c}n+k-1 \\ k\end{array}\right)$ distinct directions by solving a linear system of equations.

In the context of polygon retrieval $(n=2)$ from tomographic data in [24, 13], geometric moments and then complex moments are computed from directional moments. Complex moments can be understood as moments in the direction $\delta=(1, \mathrm{i})$. At the core of this shape-from-moments problem is Davis' integration formula for an analytic function $f$ on the polygon $\mathcal{V}$ in the complex plane $[7]$

$$
\iint_{\mathcal{V}} f^{\prime \prime}(x+\mathrm{i} y) d x d y=\sum_{v \in \mathcal{V}} a_{v} f(v)
$$

where the $v \in \mathcal{V}$ are here interpreted as complex numbers. Assuming that $\check{v}$ and $\hat{v}$ are the vertices adjacent to $v$, the coefficients in Davis' formula are

$$
a_{v}=\frac{V_{v}}{(v-\check{v})(v-\hat{v})}
$$

where $V_{v}$ is the oriented area of the parallelogram defined by the vectors with vertices $\check{v}-v$ and $\hat{v}-v$.

This formula bears a generalisation to any dimension, known as Brion's identities. The formula relates the directional moments to the projections of the vertices. This allows us to work directly with directional moments, which are data that can be deduced from tomographic measurements.

Theorem 2.1. [16] Provided that the orthogonal projections of the $r$ vertices of the convex polytope $\mathcal{V}$ on the direction $\delta$ are distinct, we have the following equalities

$$
\text { and } \begin{aligned}
\frac{(k+n) !}{k !} m_{k}(\delta) & =\sum_{v \in \mathcal{V}} a_{v}(\delta)\langle v, \delta\rangle^{n+k}, \quad k \geq 0, \\
0 & =\sum_{v \in \mathcal{V}} a_{v}(\delta)\langle v, \delta\rangle^{n-k}, \quad 1 \leq k \leq n,
\end{aligned}
$$

where the $a_{v}(\delta)$ depend on $\delta$ and the adjacent vertices of $v$ in a triangulation of $\mathcal{V}$.

Moreover

$$
a_{v}(\delta) \neq 0, \quad v \in \mathcal{V}
$$


The formula for the coefficients $a_{v}(\delta)$ is given in [2, Section 10.3] when $\mathcal{V}$ is a simple convex polytope. That is, each vertex in the polytope has exactly $n$ adjacent vertices. Let $\mathcal{V}_{v}$ be the set of $n$ adjacent vertices of $v$. The volume $V_{v}$ of the parallepiped determined by $\mathcal{V}_{v}$ is obtained through the determinant of the edge vectors of $\mathcal{V}_{v}$. Then

$$
a_{v}(\delta)=\frac{V_{v}}{\prod_{u \in \mathcal{V}_{v}}\langle v-u, \delta\rangle} .
$$

In particular, for a simplex $\triangle$ with vertices $v_{0}, v_{1}, \ldots, v_{n}$,

$$
\frac{(k+n) !}{k !} \int_{\triangle}\langle x, \delta\rangle^{k} d x=V \sum_{i=0}^{n} \frac{\left\langle v_{i}, \delta\right\rangle^{k+n}}{\prod_{j \neq i}\left\langle v_{i}-v_{j}, \delta\right\rangle}=V \sum_{k_{0}+\ldots+k_{n}=k}\left\langle v_{0}, \delta\right\rangle^{k_{0}} \ldots\left\langle v_{n}, \delta\right\rangle^{k_{n}}
$$

where $V=V_{v_{0}}=\ldots=V_{v_{n}}$. Notice that this is actually a polynomial in $\delta$ though we shall use its more compact rational expression.

For a more general convex polytope, one has to consider a partition of the polytope into simplices that does not introduce any additional vertex [2, Theorem 3.1]. The coefficients $a_{v}(\delta)$ for the convex polytope is then a sum of its sibblings in the formulae for the simplices. That they do not vanish is proved in [16].

The directions $\delta \in \mathbb{R}^{n}$ to which the theorem applies are those for which $\langle u, \delta\rangle \neq\langle v, \delta\rangle$ for all distinct $u, v \in \mathcal{V}$. Those are the generic directions. We examine what happens when $\delta$ fails to be generic in this meaning.

On the one hand, Brion's identities are correct for any $\delta$ that does not make the denominators of $a_{v}$ vanish. However, if $\delta$ is a direction for which the coefficients $a_{v}(\delta)$ are well defined but for which there are two distinct vertices $u, v \in \mathcal{V}$ such that $\langle u, \delta\rangle=\langle v, \delta\rangle$, we can write the formula with less than $r$ terms. The linear recurrence introduced in Section 3 is then of order less than $r$ and the associated Hankel matrix is of rank less than $r$.

In addition, when $\left\langle v_{0}, \delta\right\rangle=\ldots=\left\langle v_{p}, \delta\right\rangle$ for distinct vertices $v_{0}, \ldots, v_{p} \in \mathcal{V}$ that belong to the same simplex of any triangulation, there is a formula similar to (2.1) where the $p+1$ terms $\left\langle v_{i}, \delta\right\rangle^{n+k}$ are replaced by terms $\left\langle v_{0}, \delta\right\rangle^{n+k},(n+k)\left\langle v_{0}, \delta\right\rangle^{n+k-1}, \ldots,(n+k) \ldots(n+k-p+$ 1) $\left\langle v_{0}, \delta\right\rangle^{n+k-p}$. This can be deduced from the rightmost expression in (2.4). The Hankel matrix constructed in Section 3 is still of rank $r$ and admits $\left\langle v_{0}, \delta\right\rangle$ as a generalized eigenvalue of multiplicity $p+1$. See for instance $[10,23]$.

\section{Recovering the projections of the vertices}

In this section we address the problem of retrieving the projections $\mathcal{V}(\delta)=\{\langle v, \delta\rangle \mid v \in \mathcal{V}\}$ of the vertices of the convex polytope $\mathcal{V}$ from its directional moments $m_{k}(\delta)$. We recognize an inverse problem that has appeared in several areas $[12,21,25,18,24,13,16]$. While [16] approaches the problem with Prony's method, we favor a formulation in terms of generalized eigenvalues.

The standing assumption is that the projections of the vertices on the direction $\delta$ are pairwise distinct. Thus $|\mathcal{V}(\delta)|=|\mathcal{V}|=r$. Also, we assume in this section that the number of vertices is 
known. We discuss in next section how this number can be retrieved from only the knowledge of the moments.

From the directional moments $\left(m_{k}(\delta)\right)_{k}$ we introduce the sequence $\left(\mu_{k}(\delta)\right)_{k \in \mathbb{N}}$ of modified directional moments defined by

$$
\begin{array}{ll}
\mu_{k}(\delta)=0, & 0 \leq k \leq n-1, \\
\mu_{k}(\delta)=\frac{k !}{(k-n) !} m_{k-n}(\delta), \quad k \geq n .
\end{array}
$$

By Theorem 2.1 there exist $r$ non-zero real numbers $a_{v}(\delta)$ such that this sequence satisfies $\mu_{k}(\delta)=\sum_{v \in \mathcal{V}} a_{v}(\delta)\langle v, \delta\rangle^{k}, k \in \mathbb{N}$. The goal is to retrieve the $r$ elements $\langle v, \delta\rangle$ of $\mathcal{V}(\delta)$ from $\left(\mu_{k}(\delta)\right)_{k}$ and hence $\left(m_{k}(\delta)\right)_{k}$. This is just an instance of the following problem.

Inverse problem: Consider a sequence $\left(\mu_{k}\right)_{k \in \mathbb{N}}$ such that for some non-zero real (or complex) numbers $a_{1}, \ldots, a_{r}$ and pairwise distinct real (or complex) numbers $w_{1}, \ldots w_{r}$,

$$
\mu_{k}=\sum_{i=1}^{r} a_{i} w_{i}^{k}, \quad \forall k \in \mathbb{N}
$$

The problem is to find the $w_{i}$ from the knowledge of $r$ and $\left(\mu_{k}\right)_{0 \leq k \leq 2 r-1}$. It can be tackled by Prony's method recast as a generalized eigenvalue problem.

In this paper we deal with computed directional moments. In comparison to measured directional moments, we can work with a selected precision and we do not take care of noise effects in the data. When working with measured information and having $2 R-n$ moments available per direction, it is best to replace the square $r \times r$ Hankel matrices by rectangular $R \times r$ Hankel matrices and introduce Least Squares or Maximum Likelihood methods to solve this inverse problem $[9,15,25]$.

First one observes that the sequence $\left(\mu_{k}\right)_{k}$ is a solution of a recurrence equation of order $r$, namely

$$
\mu_{k+r}=p_{r-1} \mu_{k+r-1}+\ldots+p_{0} \mu_{k},
$$

where $\left(-p_{0}, \ldots,-p_{r-1}, 1\right)$ are the coefficients of the polynomial

$$
p(z)=\prod_{i=1}^{r}\left(z-w_{i}\right)=z^{r}-p_{r-1} z^{r-1}-\ldots-p_{1} z-p_{0} .
$$

Applying (3.2) to $\left(\mu_{k}\right)_{k \in \mathbb{N}}$ for $k=0, \ldots, r-1$ leads to the linear system

$$
\underbrace{\left(\begin{array}{ccccc}
\mu_{0} & \mu_{1} & \ldots & & \mu_{r-1} \\
\mu_{1} & & & . \cdot & \\
\vdots & & . \cdot & & \vdots \\
& . \cdot & & & \\
\mu_{r-1} & & \ldots & & \mu_{2 r-2}
\end{array}\right)}_{H_{r}^{(0)}}\left(\begin{array}{c}
p_{0} \\
p_{1} \\
\vdots \\
\vdots \\
p_{r-1}
\end{array}\right)=\left(\begin{array}{c}
\mu_{r} \\
\mu_{r+2} \\
\vdots \\
\vdots \\
\mu_{2 r-1}
\end{array}\right) .
$$

From $\left(\mu_{k}\right)_{0 \leq k \leq 2 r-1}$ we can retrieve the characteristic polynomial $p$ of the underlying recurrence by solving the above linear system. The sought numbers $w_{1}, \ldots, w_{r}$ are the roots of this polynomial. Theses two steps (solving the linear system and computing the roots of the entailed 
polynomial) is known as Prony's method. It was introduced in [26] and is used in the context of the shape-from-moments problem in [16, 24]. The authors of $[13,20]$ introduce a solution in terms of the generalized eigenvalues of a pencil of matrices. It is based on the following facts.

We can recast (3.3) into the matrix equality:

$$
\underbrace{\left(\begin{array}{ccccc}
\mu_{0} & \mu_{1} & \ldots & & \mu_{r-1} \\
\mu_{1} & & & . \cdot & \\
\vdots & & . \cdot & & \vdots \\
& . \cdot & & &
\end{array}\right)}_{H_{r}^{(0)}} \underbrace{\left(\begin{array}{ccccc}
0 & \ldots & \ldots & 0 & p_{0} \\
1 & \ddots & & \vdots & p_{1} \\
\mu_{r-1} & & \ldots & & \mu_{2 r-2}
\end{array}\right)}_{P}=\underbrace{\left(\begin{array}{cccccc}
\mu_{1} & \mu_{2} & \ldots & & \mu_{r} \\
\mu_{2} & & & . \cdot & \\
\vdots & & . \cdot & \\
\vdots & \ddots & \ddots & 0 & \vdots \\
0 & \ldots & 0 & 1 & p_{r-1}
\end{array}\right)}_{H_{r}^{(1)}} .
$$

The last column of the matrix on the right hand side comes from Equation (3.3), while the other columns are simply shifts of the columns in the matrix on the left hand side.

Let us introduce the following notations from [18, Section 7.5] for the matrices arising in the above equality. The $r \times r$ Hankel matrix with first row given by $\left(\begin{array}{ccc}\mu_{d} & \ldots & \mu_{r+d-1}\end{array}\right)$ is denoted by $H_{r}^{(d)}$. The companion matrix of the characteristic polynomial of the recurrence (3.2) is denoted by $P$. The matrix equality (3.4) becomes $H_{r}^{(0)} P=H_{r}^{(1)}$, and more generally we have $H_{r}^{(d)} P=H_{r}^{(d+1)}$ for $d \geq 0$.

Since $w_{1}, \ldots, w_{r}$ are the roots of $p(z)=z^{r}-p_{r-1} z^{r-1}-\ldots-p_{1} z-p_{0}$, we have

$$
\underbrace{\left(\begin{array}{cccc}
1 & w_{1} & \cdots & w_{1}^{r-1} \\
\vdots & \vdots & & \vdots \\
\vdots & \vdots & & \vdots \\
1 & w_{r} & \cdots & w_{r}^{r-1}
\end{array}\right)}_{W_{r}} \underbrace{\left(\begin{array}{cccc}
0 & \cdots & 0 & p_{0} \\
1 & \ddots & \vdots & \vdots \\
\vdots & \ddots & 0 & p_{r-2} \\
0 & \ldots & 1 & p_{r-1}
\end{array}\right)}_{P}=\underbrace{\left(\begin{array}{cccc}
w_{1} & & & 0 \\
& \ddots & & \\
& & \ddots & \\
& & & w_{r}
\end{array}\right)}_{D} \underbrace{\left(\begin{array}{cccc}
1 & w_{1} & \cdots & w_{1}^{r-1} \\
\vdots & \vdots & & \vdots \\
\vdots & \vdots & & \vdots \\
1 & w_{r} & \cdots & w_{r}^{r-1}
\end{array}\right)}_{W_{r}} .
$$

Let $D$ and $W_{r}$ be respectively the diagonal and the Vandermonde matrices defined by $w_{1}, \ldots, w_{r}$ and appearing in the above equality. The latter can thus be written $W_{r} P=D W_{r}$. The $w_{i}$ being pairwise distinct, $W_{r}$ is invertible and $P W_{r}^{-1}=W_{r}^{-1} D$. That is, $w_{1}, \ldots, w_{r}$ are the eigenvalues of $P$ and $W_{r}^{-1}$ is a matrix of eigenvectors for $P$. From $H_{r}^{(1)}=H_{r}^{(0)} P$ in (3.4) we can deduce

$$
H_{r}^{(1)} W_{r}^{-1}=H_{r}^{(0)} W_{r}^{-1} D,
$$

and more generally, $H_{r}^{(d+1)} W_{r}^{-1}=H_{r}^{(d)} W_{r}^{-1} D$, for $d \in \mathbb{N}$. Thus $w_{1}, \ldots, w_{r}$ are the generalized eigenvalues of the matrix pencils $\left(H_{r}^{(d+1)}, H_{r}^{(d)}\right)$ and $W_{r}^{-1}$ is a matrix of associated generalized eigenvectors.

Computing generalized eigenvalues is a classical problem in numerical linear algebra $[14,8$, 19]. The structured problem we consider here is unfortunately known to be potentially illconditioned. Following [4] we can give an upper bound for the conditioning of the generalized eigenvalue problem as a constant multiplied by the square of the condition number of the Vandermonde matrix $W_{r}$. 
To come back to our initial problem of retrieving $\mathcal{V}(\delta)$ from $\left(\mu_{k}(\delta)\right)_{k}$ we introduce the pencil of Hankel matrices $\left(H_{r}^{(1)}(\delta), H_{r}^{(0)}(\delta)\right)$. Its generalized eigenvalues are the elements of $\mathcal{V}(\delta)$. From those we can construct a matrix of generalized eigenvectors, given by the inverse of the Vandermonde matrix $W_{r}(\delta)$. The condition number of $\mathcal{W}_{r}(\delta)$ is denoted $\kappa(\delta)$. To reduce the conditionning of the generalized eigenvalue problem, which is of order $\kappa(\delta)^{2}$, we consider polytopes lying in the unit ball.

\section{Estimating the number of vertices}

So far, the number $r$ of vertices has been assumed to be given. But $r$ can also be an unknown of the problem. In this section, we discuss how to numerically retrieve this number from the Hankel matrices $H_{k}^{(0)}(\delta)$ formed from the sequence of modified directional moments $\left(\mu_{k}(\delta)\right)_{k}$ in a generic direction $\delta$.

One first observes that $H_{r+\ell}^{(0)}(\delta)$ is at most of rank $r$ for any $\ell \geq 0$. Indeed the sequence $\left(\mu_{k}(\delta)\right)_{k \in \mathbb{N}}$ satisfies a recurrence equation (3.1) of order $r$. For any $\ell>0$, each of the last $\ell$ columns of $H_{r+\ell}^{(0)}(\delta)$ is thus a linear combination of the previous $r$ columns. Now, noting $w_{1}, \ldots, w_{r}$ the elements of $\mathcal{V}(\delta)$, we examine the Vandermonde factorisation of the Hankel matrix:

$$
H_{k}^{(0)}(\delta)=\underbrace{\left[\begin{array}{cccc}
1 & 1 & \cdots & 1 \\
w_{1} & w_{2} & \cdots & w_{r} \\
\vdots & \vdots & \ddots & \vdots \\
w_{1}^{k-1} & w_{2}^{k-1} & \cdots & w_{r}^{k-1}
\end{array}\right]}_{{ }^{t} W_{k}(\delta)} \underbrace{\left[\begin{array}{ccccc}
a_{1}(\delta) & 0 & \cdots & 0 \\
0 & a_{2}(\delta) & \ddots & \vdots \\
\vdots & \ddots & \ddots & 0 \\
0 & \cdots & 0 & a_{r}(\delta)
\end{array}\right]}_{A(\delta)} \underbrace{\left[\begin{array}{cccc}
1 & w_{1} & \cdots & w_{1}^{k-1} \\
1 & w_{2} & \cdots & w_{2}^{k-1} \\
\vdots & \vdots & \ddots & \vdots \\
1 & w_{r} & \cdots & w_{r}^{k-1}
\end{array}\right]}_{W_{k}(\delta)} .
$$

For a generic direction $\delta$, the $r$ elements $w_{i}$ of $\mathcal{V}(\delta)$ are pairwise distinct and therefore $\operatorname{det} H_{r}^{(0)}(\delta) \neq$ 0 . It follows that $H_{r+\ell}^{(0)}(\delta)$ is exactly of rank $r$, for all $\ell \geq 0$.

Based on this observation, if a strict upper bound $R$ for the number of vertices $r$ is given, then $r$ can be determined as the rank of $H_{R}^{(0)}(\delta)$. A caveat is that this matrix may be ill-conditioned. The condition number of $H_{r}^{(0)}(\delta)$ is determined by the condition number of $W_{r}(\delta)$ and $A(\delta)$ in (4.1). For this we also refer to the discussion in [12] and [22] that examines the situation in the context of sparse interpolation. The condition number of the Vandermonde matrix $W_{r}(\delta)$ depends on the distribution of the numbers in $\mathcal{V}(\delta)$ of the projections of the vertices in the direction $\delta$ [11]. As for the matrix $A(\delta)$, having one of the $a_{i}(\delta)$ too small can also lead to an incorrect (numerical) rank for $H_{R}^{(0)}$. Since we can (even randomly) select multiple directions for the projections, we can retain only those directions for which not both $W_{r}(\delta)$ and $A(\delta)$ are too ill-conditioned. Alternatively we could apply the rank estimates for Hankel matrices of [5].

Therefore, if we have an overestimation $R$ of the number of vertices we can recover the exact number from the analysis of the numerical rank of $H_{R}^{(0)}(\delta)$. In practice we analyze the singular values of $H_{R}^{(0)}(\delta)$ computed by a Singular Value Decomposition [14, 8]. This is discussed on specific cases in Section 6 and 7. 


\section{Reconstruction of the vertices from their projections}

In this section we show how to retrieve the vertices $v$ from the sets of projections $\mathcal{V}(\delta)=$ $\{\langle v, \delta\rangle, v \in \mathcal{V}\}$ for $\delta \in \Delta$, where $\Delta$ is a set of $s>n$ vectors in the unit sphere $S_{n}$. We have at our disposal $s$ sets $\mathcal{V}(\delta)$ each containing $r$ distinct vertex projections,

$$
\mathcal{V}(\delta)=\{\langle v, \delta\rangle: v \in \mathcal{V}\}, \quad \delta \in \Delta, \quad\|\delta\|=1, \quad \# \Delta=s>n .
$$

For each $\delta \in \Delta, \mathcal{V}(\delta)$ was obtained from the moments $\left(m_{k}(\delta)\right)_{0 \leq k \leq 2 r-n-1}$, as discussed in Section 3 .

The problem is that we do not know which projection in $\mathcal{V}(\delta)$ belongs to which vertex. So we need to find the correct labelling of the projections concurrently with the computation of the vertex coordinates.

Another issue is that the vertex projections are computed from a generalized eigenvalue problem that may not have been perfectly conditioned. So we need to take some tolerance into account when interpreting the projections and using them in subsequent computations.

Let the convex polytope be contained in a Euclidean unit ball. So all projections $w=\langle v, \delta\rangle$ be bounded by

$$
|w|=|\langle v, \delta\rangle| \leq 1
$$

We assume that the computed values for all $w$ are in error by at most $\epsilon>0$. With this error bound $\epsilon$ we define intervals

$$
\mathcal{W}(v, \delta)=[w-\epsilon, w+\epsilon]=\left[w^{-}(v, \delta), w^{+}(v, \delta)\right], \quad w=\langle v, \delta\rangle .
$$

The bound $\epsilon$ is of the order of $\kappa\left(W_{r}^{2}\right)$ multiplied by the bound on the inaccuracy of the computed directional moments $\tilde{\mu}$, in other words,

$$
\epsilon=\mathrm{O}\left(\kappa\left(W_{r}^{2}\right) \frac{\tilde{\mu}_{k}(\delta)-\mu_{k}(\delta) \mid}{\left|\mu_{k}(\delta)\right|}\right), \quad k=0,1, \ldots, 2 r-1 .
$$

We remark at this point that the computation can only continue if for at least $n+1$ directions $\delta$ the intervals $\mathcal{W}(v, \delta)$ are disjoint. For simplicity, we assume in the sequel that in all $s$ directions the intervals are disjoint.

An algorithm for the reconstruction of the vertex coordinates can now be based on the computation of $r$ linear interval interpolants in $n$ variables, of the form

$$
q_{v}(\delta)=\langle v, \delta\rangle, \quad \delta \in S_{n},
$$

where the interpolation conditions for each $q_{v}(\delta)$ are, that $q_{v}(\delta) \in \mathcal{W}(v, \delta)$ for all $\delta \in \Delta$. Each of the $r$ functions $q_{v}(\delta)$ interpolates only one $\mathcal{W}$-interval per $\mathcal{V}(\delta)$ but $s$ of those values across the sets $\mathcal{V}(\delta)$. For a graphical illustration in the case $n=2$ we refer to Figure $1(\mathrm{~d})$. In the end, with $\delta=\left(\delta_{1}, \ldots, \delta_{n}\right)$ being the variables in each of the $r$ interpolation problems

$$
q_{v}(\delta)=\sum_{i=1}^{n} u_{i}^{(j)} \delta_{i} \in \mathcal{W}(v, \delta), \quad \delta \in \Delta, \quad j=1, \ldots, r,
$$

the coefficients $u_{i}^{(j)}$ in the linear form are the coordinates of one vertex $v \in \mathcal{V}$. So

$$
v_{j}=\left(u_{1}^{(j)}, \ldots, u_{n}^{(j)}\right), \quad j=1, \ldots, r .
$$


We remark at this point that any set of $n$ projections can be interpolated by a function of the form $q_{v}(\delta)$, even if the width of the intervals is zero. At the same time any combination of $s$ projections can be approximated in the least squares sense by a function of the form $q_{v}(\delta)$. So none of these classical approaches is very useful in figuring out which projections belong to the same vertex $v$. But an interval interpolant through at least $n+1$ disjoint intervals does the job: the nonzero interval width compensates for overdetermining the linear interpolant by at least one interpolation condition. The interval interpolant is stringing the intervals, containing the projections, like beads on the graph of the interpolating function. Any interval, meaning any projection, through which it passes, is marked as belonging to the same vertex.

Naturally, an interval interpolation problem does not need to have a unique solution: sufficiently small perturbations of the coefficients in the linear form may not violate the interval interpolation conditions. In [27] is described how the most robust interval interpolant of the form given in (5.2) is computed. By this we mean the interpolant that stays away as far as possible from violating the interpolation conditions imposed by the interval bounds. This interpolant is unique and its computation is based on the computation of Chebyshev centers.

Because of the labelling problem of the projections $w$, the interval interpolation algorithm becomes a 2 -step procedure. In a first step we take the $n$ best conditioned sets $\mathcal{V}(\delta)$ and check which combination of $w$-values, one taken from each of the $n$ best conditioned $\mathcal{V}(\delta)$, delivers a (non-interval) interpolant

$$
\sum_{i=1}^{n} v_{i} \delta_{i}=\langle v, \delta\rangle, \quad \delta \in \hat{\Delta} \subset \Delta, \quad \# \hat{\Delta}=n
$$

that additionally intersects one interval $\mathcal{W}(v, \delta)$ at each of the $s-n$ remaining sets $\mathcal{V}(\delta)$. Only linear interpolants (5.3) that intersect these other $s-n$ intervals (and hence all $s$ intervals) reflect a correct labelling of the projections. These better conditioned $\mathcal{V}(\delta)$ are usually near to one another.

Since in this first step the coordinates of $v$ are computed from only $n$ of the $s$ available directions, the obtained values are not maximally accurate. After rearranging the $s$ sets $\mathcal{V}(\delta)$, each containing $r$ projections, into $r$ sets (organized per vertex) of $s$ projections, the vertex coordinates can be computed to maximal accuracy from the total of its $s$ projections. To this end the interval interpolation method can be continued with the computation of the most robust interval interpolant satisfying (5.2).

In order to make things easier to understand we now go over the 2-step procedure for the reconstruction of a polygon $(n=2)$ with $r$ vertices, illustrated in Figure 1(d).

Let us have at our disposal $s$ sets $\mathcal{V}(\delta)$ with $\delta=(\cos \theta, \sin \theta)$. Each set $\mathcal{V}(\delta)$ contains the $r$ projections $\langle v, \delta\rangle=u_{1} \cos \theta+u_{2} \sin \theta$ of the vertices, but unordered. To find out which projections in different $\mathcal{V}(\delta)$ belong to the same vertex, we select two nearby directions $\delta_{1}$ and $\delta_{2}$ for which the computations are sufficiently well-conditioned, and we start pairing up the projections in $\mathcal{V}\left(\delta_{1}\right)$ and $\mathcal{V}\left(\delta_{2}\right)$, for instance according to proximity because of the continuity of $q_{v}(\delta)$. In this first step, the worst case scenario is that we have to consider all combinations to find the correct match. But working with nearby directions reduces this combinatorial aspect with high probability.

Then we compute the interpolants (5.2) one by one ( $r$ in total) and check for each of them whether it also interpolates, and which values it interpolates at the remaining $s-2$ sets $\mathcal{V}(\delta)$. 
Only after having discovered the correct matches of the different projections we can compute the best interval interpolants (5.2) and extract the coordinates of the different vertices.

For the second step we apply the technique developed in [27]. For each $v$, the input is the set of projections $\langle v, \delta\rangle$ for various directions $\delta$. The output are robust $\left(u_{1}, \ldots, u_{n}\right) \in \mathbb{R}^{n}$ the coordinates of the vertex $v$. They are obtained by solving the following linear interval interpolation problem. We look for the coefficients of a linear function $q_{v}: S_{n} \rightarrow \mathbb{R}$ such that

$$
q_{v}(\delta)=\langle v, \delta\rangle=u_{1} \delta_{1}+\ldots+u_{n} \delta_{n}
$$

and

$$
\left|w(v, \delta)-q_{v}(\delta)\right| \leq \epsilon, \quad \delta \in \Delta
$$

\section{Simulations}

We now illustrate the proposed approach for the reconstruction of polytopes from their directional moments. For our simulations we consider centered and scaled polytopes: The origin is the center of gravity of the polytope and the vertices lie in the Euclidean unit ball. This geometric normalisation corresponds to a transformation on the moments as described in [13].

The vertices of the polytope are to be reconstructed from directional moments. The latter are typically computed from measurements, as for instance in tomography. The proposed reconstruction of a convex polytope in dimension $n$ requires directional moments in at least $n+1$ generic directions. The order of the moments required in each direction then depends on the number $r$ of vertices: we need directional moments up to order $2 r-n-1$. In dimension 2 this can be compared to [24], where the complex moments up to order $2 r-3$ are obtained from directional moments up to the same order in $2 r-2$ directions.

For a given direction $\delta$, the directional moments $m_{k}(\delta)$ are used to form the entries of the pair of Hankel matrices $\left(H_{r}^{(1)}, H_{r}^{(0)}\right)$ described in Section 3. The algorithm consists of 3 main steps:

1. Determine the number $r$ of vertices by analyzing the singular values of the Hankel matrix $H_{R}^{(0)}$ for $R>r$ big enough and a few number of random directions $\delta$, as described in Section 4 .

2. Compute the generalized eigenvalues for the pair of matrices $\left(H_{r}^{(1)}, H_{r}^{(0)}\right)$ in at least $n+1$ directions as in Section 3. Determine the condition number $\kappa\left(W_{r}\right)$ of the Vandermonde matrix $W_{r}$ formed by those generalized eigenvalues. The generalized eigenvalues are the projections of the vertices of the polytope with an error determined by a multiple of $\kappa\left(W_{r}\right)^{2}$.

3. Recover the vertices $\mathcal{V}$ from their approximate projections using the interval interpolation technique described in Section 5.

The first two steps are performed using standard numerical linear algebra routines from the NAG library in Maple. In particular, Step 1 makes use of the singular value decomposition and Step 2 of the QZ-algorithm [29]. Step 3 is implemented in Matlab. Every computations in this Section are performed in double precision. 
In the second step, we sample a number of directions and retain those for which the condition number is the smallest. The condition number is indeed dependent on the direction : $\kappa\left(W_{r}\right)$ depends on the distribution of the projected vertices [11]. In particular it increases when the projections of two vertices get closer to one another.

Furthermore, to cut down on the combinatorial complexity of the interpolation scheme in the last step, it makes sense to select directions reasonably close to one another. We make use of a generic reference direction $\hat{\delta}$ with reasonable conditioning and other directions in the neighborhood.

\subsection{Reconstruction of polygons}

We begin our simulations with the reconstruction of 2-dimensional polygons. A direction $\delta=$ $(\cos \theta, \sin \theta)$ is represented by an angle $\left.\theta \in]-\frac{\pi}{2}, \frac{\pi}{2}\right]$. The projection of vertex $v_{i}=\left(x_{i}, y_{i}\right)$ is given by $w_{i}=x_{i} \cos \theta+y_{i} \sin \theta$. Then the interval interpolation problem formulated in terms of $\theta$ is

$$
x_{i} \cos \theta+y_{i} \sin \theta \in\left[w_{i}^{-}, w_{i}^{+}\right], \quad 1 \leq i \leq r,
$$

where $w_{i}^{-}$and $w_{i}^{+}$are as in (5.1).

\subsubsection{Reconstruction of a regular hexagon}

The regular hexagon $(r=6)$ is presented in Figure 1(a). The condition number $\kappa\left(W_{6}\right)$ increases drastically when we approach the axes of symmetry (arrows in Figure 1(a)). In Figure 1(b) the condition number $\kappa\left(W_{6}\right)$ of the Vandermonde matrix $W_{6}$ is plotted for 300 equidistant generic directions. The condition number is minimal for directions bisecting two consecutive symmetry axes, represented by dotted lines in Figure 1(a).

The moments $\mu_{k}$ are computed in double precision. The number $r$ of vertices is retrieved as the rank of $H_{k}^{(0)}$, for $k$ large enough. The singular values of $H_{7}^{(0)}$ are plotted in Figure 1(c) for three directions picked at random.

We choose the direction $\hat{\delta}$ with angle $\hat{\theta}=\frac{5 \pi}{12}$. It corresponds to one of the bisectors. We then take 4 nearby directions $\hat{\theta} \pm 0.05, \hat{\theta} \pm 0.10$. For each of the 5 directions we construct the pair of Hankel matrices $\left(H_{6}^{(1)}, H_{6}^{(0)}\right)$ and compute their generalized eigenvalues. For these directions all $\kappa\left(W_{6}\right)$ are around 300. We take $w^{+}(v, \delta)-w^{-}(v, \delta)=2 \times 10^{-10}$ in (5.1) with $|\langle v, \delta\rangle| \leq 1$.

We compute the interval interpolants from the 5 sets of projections $\mathcal{V}(\hat{\delta}+k 0.05), k=-2,-1$, $0,1,2$. The relative error on the computed projections is bounded overall by $6.4 \times 10^{-13}$ for the 5 chosen directions. The coordinates of the 6 different vertices of the regular hexagon are recovered as the coefficients of the interpolants graphed in Figure 1(d). The relative error on each computed coordinate compared to its true value is bounded overall by $1.5 \times 10^{-12}$.

\subsubsection{Reconstruction of a polygon with 12 vertices}

In this second simulation, we consider a random 12-gon, centered and scaled. It is drawn in Figure 2(a). 

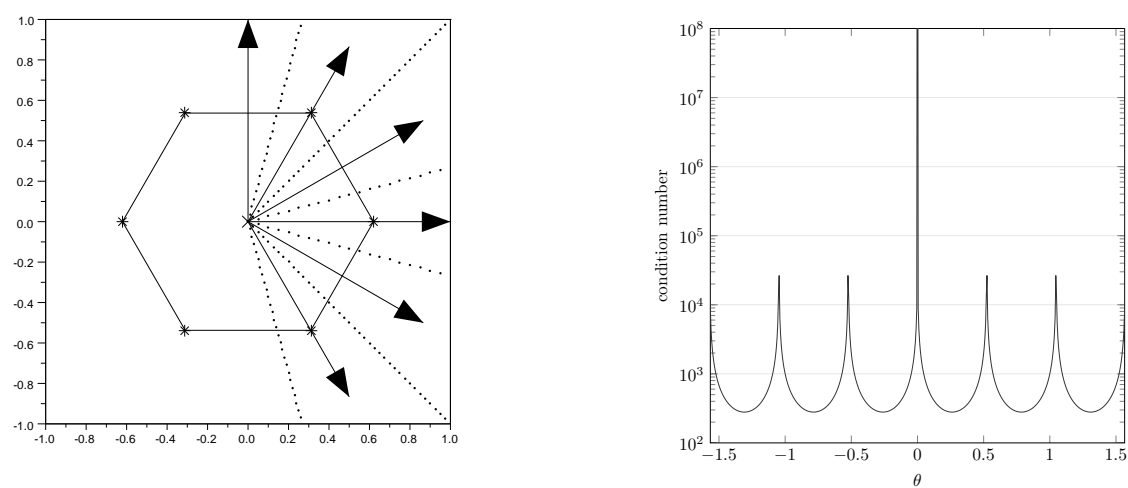

(a) Regular hexagon, its axes of symmetry and their (b) Condition number $\kappa\left(W_{6}\right)$ with bisectors respect to the direction $(\cos \theta, \sin \theta)$

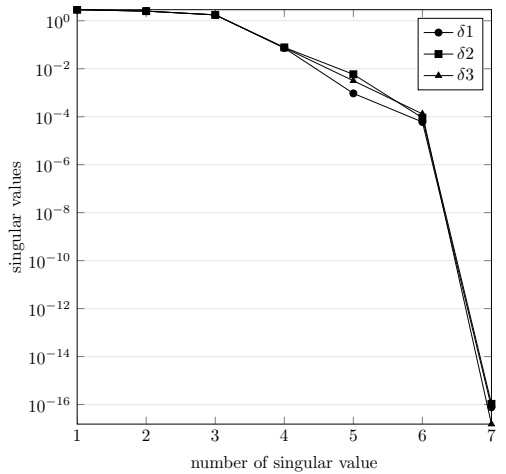

(c) Singular values of $H_{7}^{(0)}$

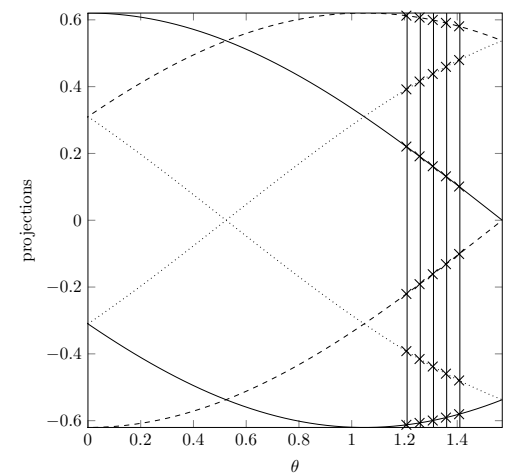

(d) Interpolation with intervals of width $2 \times 10^{-10}$

Figure 1: Regular hexagon

As in the case of the hexagon above, the number of vertices is retrieved by computing the singular values of $H_{15}^{(0)}$ in 3 directions. From Figure 2(b) we deduce that the numerical rank is $r=12$.

After inspecting some directions, we choose the reference direction $\hat{\delta}$ with $\hat{\theta}=0.379521$ (arrow in Figure 2(a)) and 4 other nearby directions $\hat{\theta} \pm 0.05, \hat{\theta} \pm 0.10$. The projections of the vertices on these directions are obtained as the generalized eigenvalues of the pairs of Hankel matrices $\left(H_{12}^{(1)}, H_{12}^{(0)}\right)$ whose entries are obtained from the respective directional moments up to order 21. The condition number $\kappa\left(W_{12}\right)$ of the matrix of generalized eigenvectors is for all 5 directions around $7 \times 10^{5}$. The relative error on the computed projections compared to their true values is bounded by $1.8 \times 10^{-7}$.

From these 5 sets $\mathcal{V}(\hat{\delta}-0.10), \mathcal{V}(\hat{\delta}-0.05), \mathcal{V}(\hat{\delta}), \mathcal{V}(\hat{\delta}+0.05), \mathcal{V}(\hat{\delta}+0.10)$, we compute the 12 linear interval interpolants. We take $w^{+}(v, \delta)-w^{-}(v, \delta)=2 \times 10^{-5}$ in (5.1). The relative error in each coordinate is bounded by $3.9 \times 10^{-6}$.

Note that even the two very close vertices in the top right corner in Figure 2(a) are recovered with the accuracy mentioned above. The distance between them is only of the order of $10^{-2}$. 


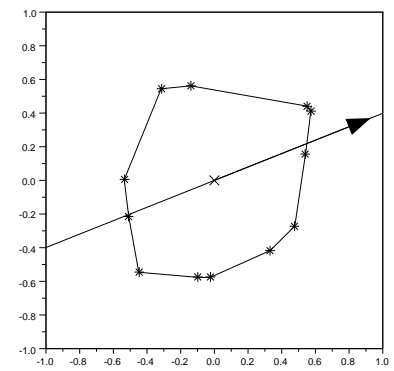

(a) Centered and scaled polygon

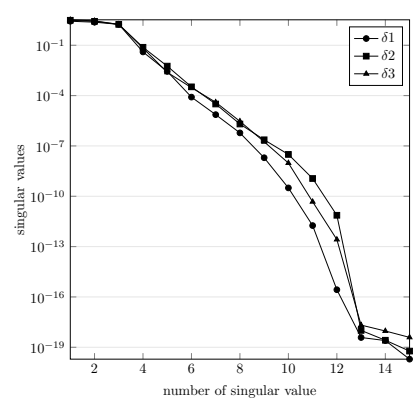

(b) Singular values of $H_{15}^{(0)}$

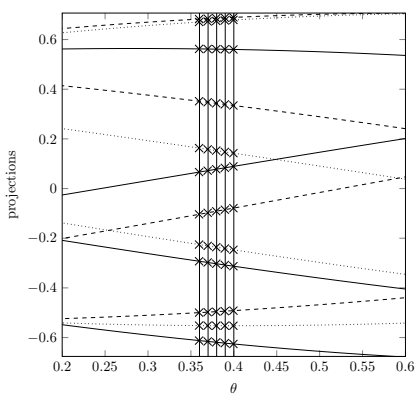

(c) Interpolation with intervals of width $2 \times 10^{-5}$

Figure 2: Polygon with 12 vertices

\subsection{Reconstruction of polyhedra}

We now consider the reconstruction of convex polyhedra in dimension 3. The dimension does not introduce new difficulties in our method. A direction $\delta$ is represented by a unit vector $(\cos (\theta) \cos (\phi), \cos (\theta) \sin (\phi), \sin (\theta))$ with $(\theta, \phi)$ lying in $\left.\left.\left.]-\frac{\pi}{2}, \frac{\pi}{2}\right] \times\right]-\frac{\pi}{2}, \frac{\pi}{2}\right]$. The projection of vertex $v_{i}=\left(x_{i}, y_{i}, z_{i}\right)$ on $\delta$ equals $w_{i}=x_{i} \cos (\theta) \cos (\phi)+y_{i} \sin (\theta) \cos (\phi)+z_{i} \sin (\phi)$. The coordinates $x_{i}, y_{i}, z_{i}$ are the unknowns in the linear interval interpolation problem

$$
w_{i} \in\left[w_{i}^{-}, w_{i}^{+}\right], \quad 1 \leq i \leq r .
$$

\subsubsection{Reconstruction of a polyhedron with well-distributed vertices}

We first consider a polyhedron with 10 vertices represented in Figure 3(a).

We retrieve the number of vertices of the polyhedron by computing the numerical rank of the Hankel matrix $H_{11}^{(0)}$ in 3 different directions. The singular values of $H_{11}^{(0)}$ are plotted in Figure 3(b) for three random directions.

After inspecting several directions, we select $(\hat{\theta}, \hat{\phi})=(-1.256637,0.261799)$ for the reference direction $\hat{\delta}$ and 4 other nearby directions where the condition number $\kappa\left(W_{10}\right)$ is of the order of $10^{4} . \hat{\delta}$ is indicated by an arrow and a dotted line in Figure $3(\mathrm{a})$. We take the nearby directions as $(\hat{\theta}+\psi, \hat{\phi}),(\hat{\theta}, \hat{\phi}-\psi),(\hat{\theta}-\psi, \hat{\phi}+\psi)$ and $(\hat{\theta}+\psi, \hat{\phi}+\psi)$ with $\psi=0.01$. For each direction, the pair of matrices $\left(H_{10}^{(1)}, H_{10}^{(0)}\right)$ is built with directional moments up to order 16 . The generalized eigenvalues of the pairs $\left(H_{10}^{(1)}, H_{10}^{(0)}\right)$ provide the projections of the vertices with a relative error bounded by $1.3 \times 10^{-8}$.

We compute the 10 linear interval interpolants using intervals of width $2 \times 10^{-7}$ for the projections. In Figure 3(c) we show one of those surfaces. The coordinates of the 10 vertices of the polyhedron are obtained as the coefficients of the interpolants. The relative error on this final result is bounded by $8.3 \times 10^{-7}$. 


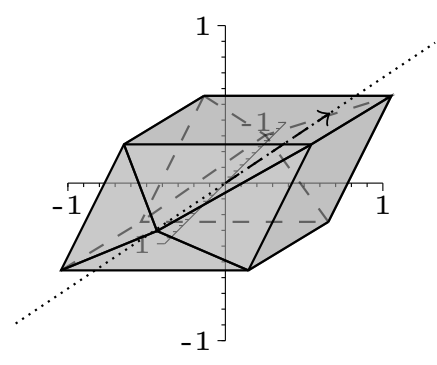

(a) Polyhedron

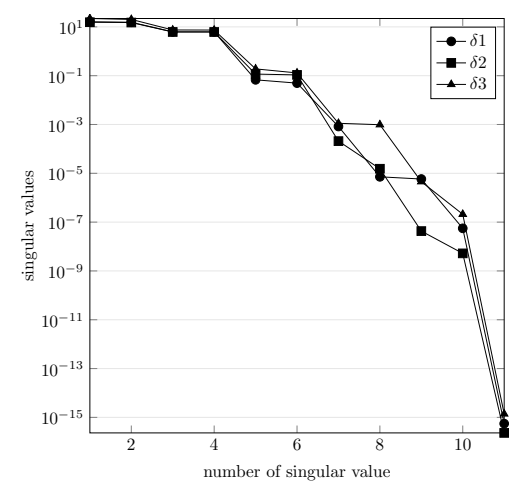

(b) Singular values of $H_{11}^{(0)}$

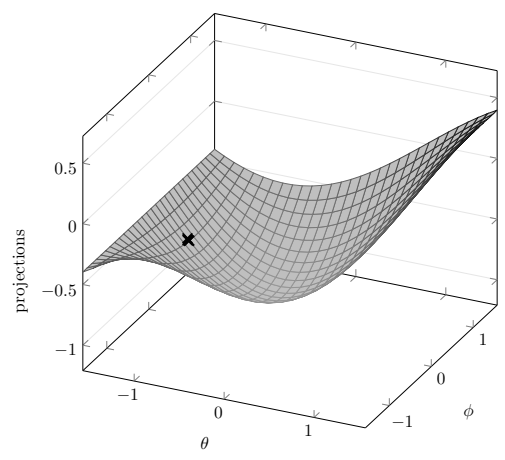

(c) Interpolant for one vertex

Figure 3: First polyhedron with 10 vertices

\subsubsection{Reconstruction of a polyhedron with close vertices}

Here we examine a polyhedron with 10 vertices and a triangular face of relatively small size (see the top of Figure 4(a)).

In Figure 4(b) we plot the singular values of $H_{12}^{(0)}$ for three random directions. The gap between the tenth and the eleventh singular value appears more clearly in some of the directions.

We choose the reference direction $(\hat{\theta}, \hat{\phi})=(-0.994838,-0.994838)$ for which the condition number $\kappa\left(W_{10}\right)$ is $4.2 \times 10^{4}$. It is indicated in Figure $4(\mathrm{a})$ by an arrow and a dotted line. We additionally pick the nearby directions $(\hat{\theta}+\psi, \hat{\phi}),(\hat{\theta}, \hat{\phi}-\psi),(\hat{\theta}-\psi, \hat{\phi}+\psi)$ and $(\hat{\theta}+\psi, \hat{\phi}+\psi)$ with $\psi=0.01$. For each direction the pair of matrices $\left(H_{10}^{(1)}, H_{10}^{(0)}\right)$ is built with directional moments up to order 16 . The projections of the vertices on those directions are retrieved as the generalized eigenvalues of $\left(H_{10}^{(1)}, H_{10}^{(0)}\right)$ with a relative error bounded by $2.2 \times 10^{-7}$.

We determine the 10 interpolants for our sets of projections using intervals of width $2 \times 10^{-6}$. The coordinates of the 10 vertices appear as the coefficients of the interpolants. The relative error on these is bounded by $1.5 \times 10^{-6}$.

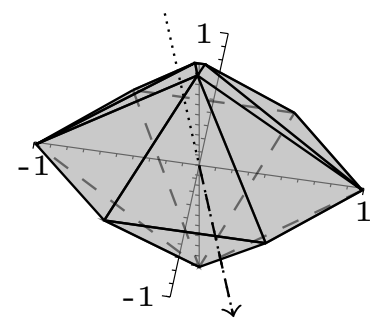

(a) Centered and scaled polyhedron

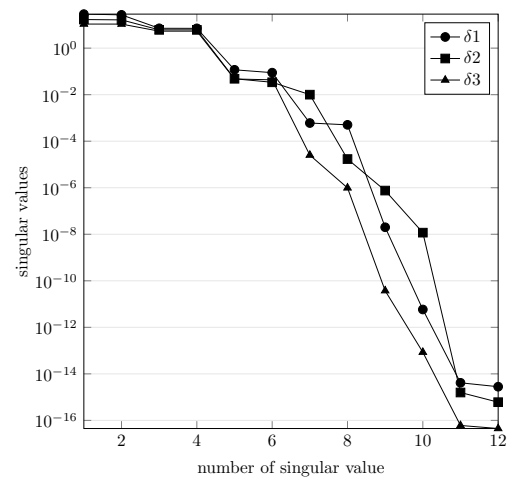

(b) Singular values of $H_{12}^{(0)}$

Figure 4: Second polyhedron with 10 vertices 


\section{Diamond}

As a challenge we choose an actual brilliant cut of a diamond. It is given as a convex polyhedron with 57 vertices and represented in Figure 5. The stone's girdle consists of pairs of vertices very close to one another. The number of vertices and the small distance between the projections of the vertices severely impact the condition number $\kappa\left(W_{r}\right)$ : double precision is no longer enough to retrieve sufficiently accurate values for the projections. We rely on the software floats of Maple to provide the needed number of digits for the computations.

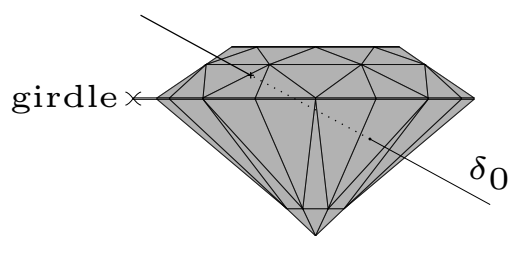

(a) Side view

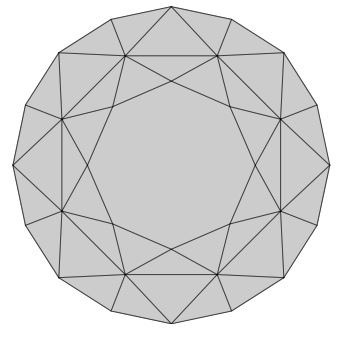

(b) Top view

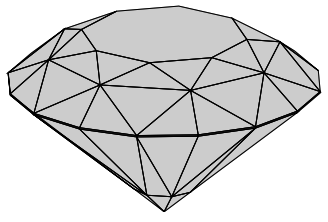

(c) In perspective

Figure 5: Diamond and reference direction $\delta_{0}$

To reliably retrieve the number of vertices, we use a precision of 70 digits. Figure 6 tracks the singular values of $H_{65}^{(0)}$ for 8 random directions and in different computational precisions.

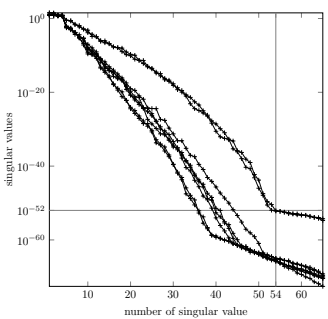

(a) 50 digits

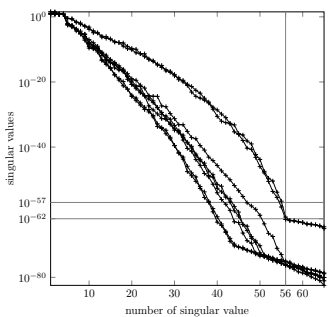

(b) 60 digits

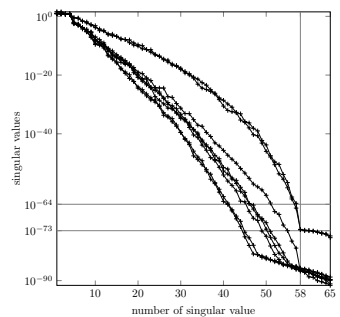

(c) 70 digits

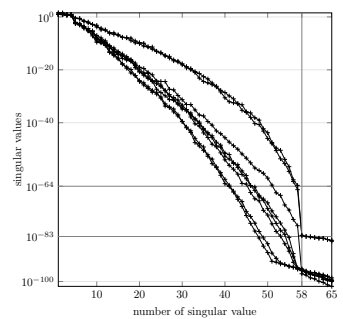

(d) 80 digits

Figure 6: Singular Values of $H_{65}^{(0)}$ for several computational precision

After sampling a rather large number of directions, we select the reference direction $(\hat{\theta}, \hat{\phi})=$ $(0.261799,1.047198)$ shown in Figure $5(\mathrm{a})$. The condition number $\kappa\left(W_{57}\right)$ for this direction is $1.67 \times 10^{33}$. We choose 4 nearby directions with a similar condition number, $(\hat{\theta}+\psi, \hat{\phi}),(\hat{\theta}, \hat{\phi}-\psi)$, $(\hat{\theta}-\psi, \hat{\phi}+\psi),(\hat{\theta}+\psi, \hat{\phi}+\psi)$ where $\psi=0.0001$. Computing with 70 digits we expect to retrieve the projections of the vertices with an accuracy of at least $10^{-3}$.

For each of the 5 selected directions $\delta$, the pair of matrices $\left(H_{57}^{(1)}, H_{57}^{(0)}\right)$ is built with the directional moments up to order 110 . The projections of the vertices are obtained as the generalized eigenvalues. The relative error is bounded by $8.1 \times 10^{-8}$.

We compute the 57 interpolants in double precision using intervals of width $2 \times 10^{-4}$. The coordinates of the vertices are the coefficients in those interpolants. The relative error is bounded by $7.8 \times 10^{-5}$. We plot the error for all the vertices in Figure 7 . 


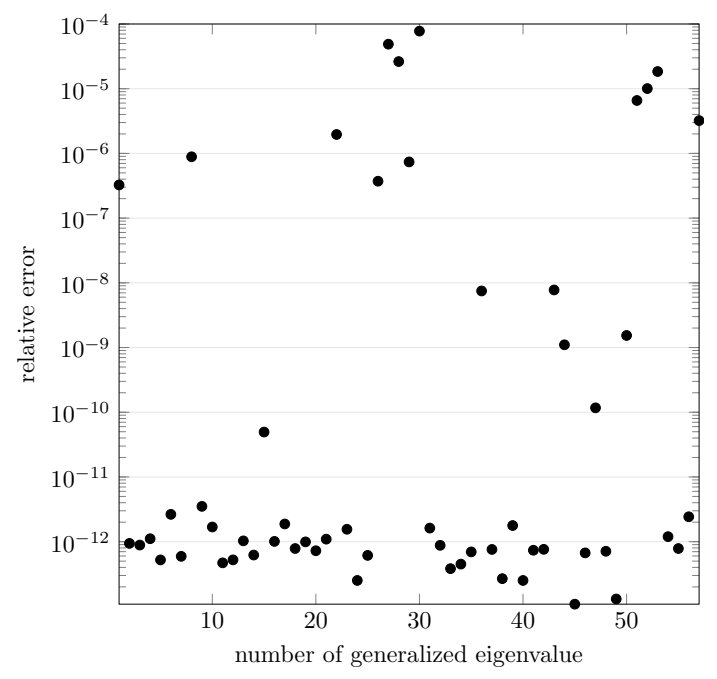

Figure 7: Error observed when operating with 70 digits

In Figure 8 we report on the error using various computational precisions. For computations with less than 65 digits we do not recover all the projections while the complete set of coordinates of the vertices can be retrieved only if we use at least 70 digits.

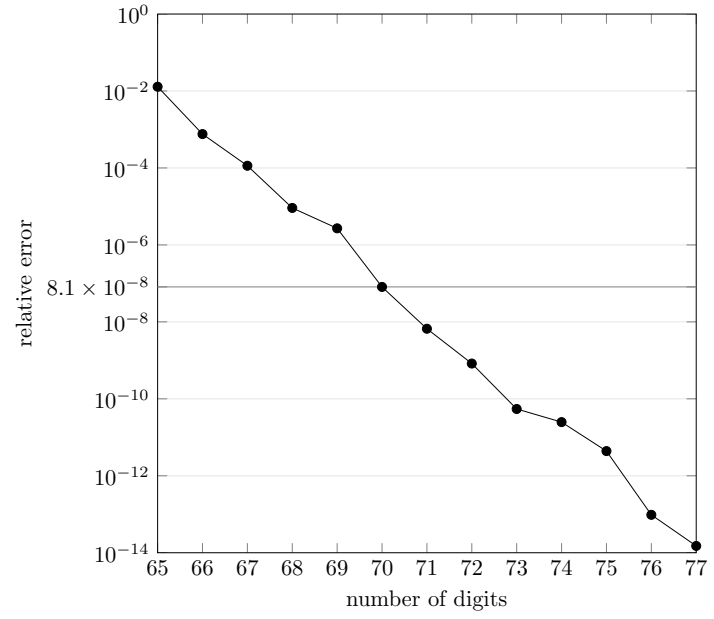

(a) Error on the retrieved projections

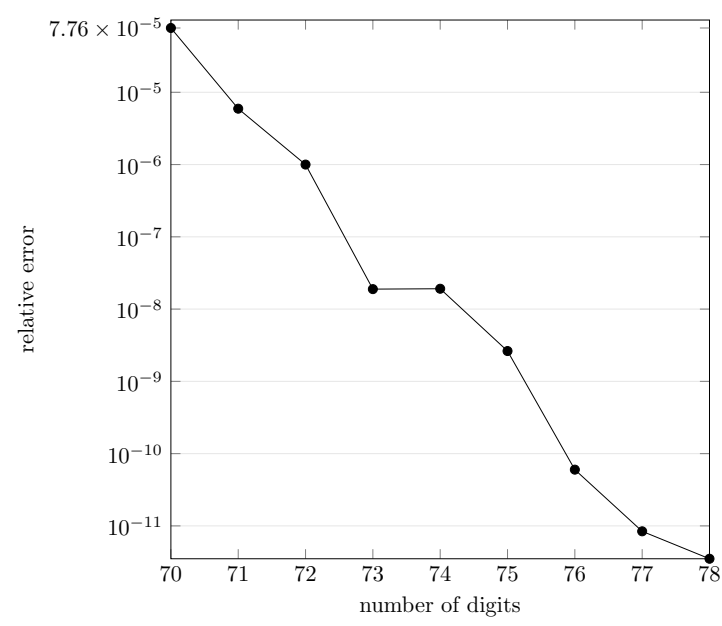

(b) Error on the retrieved vertex coordinates

Figure 8: Error for different computational precisions

\section{References}

[1] V. Baldoni, N. Berline, J. A. De Loera, M. Köppe, and M. Vergne. How to integrate a polynomial over a simplex. Math. Comp., 80(273):297-325, 2011.

[2] M. Beck and S. Robins. Computing the continuous discretely. Undergraduate Texts in Mathematics. Springer, New York, 2007. 
[3] B. Beckermann. The condition number of real Vandermonde, Krylov and positive definite Hankel matrices. Numer. Math., 85:553-577, 2000.

[4] B. Beckermann, G. H. Golub, and G. Labahn. On the numerical condition of a generalized Hankel eigenvalue problem. Numer. Math., 106(1):41-68, 2007.

[5] S. Cabay and R. Meleshko. A weakly stabel algorithm for padé approximants and the inversion of hankel matrices. SIAM Journal on Matrix Analysis and Applications, 14(3):735-765, 1993.

[6] A. Cuyt, G. Golub, P. Milanfar, and B. Verdonk. Multidimensional integral inversion, with applications in shape reconstruction. SIAM J. Sci. Comput., 27:1058-1070, 2005.

[7] P. J. Davis. Triangle formulas in the complex plane. Math. Comp., 18:569-577, 1964.

[8] J. W. Demmel. Applied numerical linear algebra. Society for Industrial and Applied Mathematics (SIAM), Philadelphia, PA, 1997.

[9] M. Elad, P. Milanfar, and G. H. Golub. Shape from moments - an estimation theory perspective. IEEE Trans. Signal Process., 52(7):1814-1829, 2004.

[10] S. Feldmann and G. Heinig. Vandermonde factorization and canonical representations of block hankel matrices. Linear Algebra and its Applications, 241-243(0):247 - 278, 1996.

[11] W. Gautschi. Optimally conditioned Vandermonde matrices. Numer. Math., 24:1-12, 1975.

[12] M. Giesbrecht, G. Labahn, and W.-s. Lee. Symbolic-numeric sparse interpolation of multivariate polynomials. J. Symbolic Comput., 44(8):943-959, 2009.

[13] G. H. Golub, P. Milanfar, and J. Varah. A stable numerical method for inverting shape from moments. SIAM J. Sci. Comput., 21(4):1222-1243, 1999/00.

[14] G. H. Golub and C. F. Van Loan. Matrix computations, volume 3 of Johns Hopkins Series in the Mathematical Sciences. Johns Hopkins University Press, Baltimore, MD, 1983.

[15] Gene Golub and Victor Pereyra. Separable nonlinear least squares: the variable projection method and its applications. Inverse Problems, 19(2):R1-R26, 2003.

[16] N. Gravin, J. Lasserre, D. V. Pasechnik, and S. Robins. The Inverse Moment Problem for Convex Polytopes. Discrete Comput. Geom., 48(3):596-621, 2012.

[17] B. Gustafsson, C. He, P. Milanfar, and M. Putinar. Reconstructing planar domains from their moments. Inverse Problems, 16(4):1053-1070, 2000.

[18] P. Henrici. Applied and computational complex analysis. Vol. 1. Wiley Classics Library. John Wiley \& Sons Inc., New York, 1988. Power series - integration-conformal mappinglocation of zeros, Reprint of the 1974 original, A Wiley-Interscience Publication.

[19] D. J. Higham and N. J. Higham. Structured backward error and condition of generalized eigenvalue problems. SIAM J. Matrix Anal. Appl., 20(2):493-512, 1999.

[20] Y. Hua and T. K. Sarkar. Matrix pencil method for estimating parameters of exponentially damped/undamped sinusoids in noise. IEEE Trans. Acoustics, Speech, and Signal Processing, 38(5):814-824, 1990. 
[21] E. Kaltofen and W.-s. Lee. Early termination in sparse interpolation algorithms. J. Symbolic Comput., 36(3-4):365-400, 2003.

[22] E. L. Kaltofen, W.-s. Lee, and Z. Yang. Fast estimates of hankel matrix condition numbers and numeric sparse interpolation. In $S N C^{\prime} 11$, pages 130-136. ACM Press, New York, NY, 2011.

[23] W.-s. Lee. From quotient-difference to generalized eigenvalues and sparse polynomial interpolation. In $S N C^{\prime} 07$, pages 110-116. ACM, New York, 2007.

[24] P. Milanfar, G. Verghese, C. Karl, and A. Willsky. Reconstructing polygons from moments with connections to array processing. IEEE Transactions on Signal Processing, 43:432-443, 1995.

[25] V. Pereyra and G. Scherer, editors. Exponential Data Fitting and its Applications. Bentham e-books, http://www. benthamscience.com/ebooks/9781608050482, 2010.

[26] C. (Baron de Prony) Riche. Essai expérimental et analytique sur les lois de la dilatabilité des fluides élastique et sur celles de la force expansive de la vapeur de l'eau et de la vapeur de l'alkool, à différentes températures. J. de l'École Polytechnique, 1:24-76, 1795.

[27] O. Salazar Celis, A. Cuyt, and B. Verdonk. Rational approximation of vertical segments. Numer. Algorithms, 45:375-388, 2007.

[28] S. Sheynin and A. Tuzikov. Explicit formulae for polyhedra moments. Pattern Recognition Letters, 22:1103-1109, 2001.

[29] J. H. Wilkinson. Kronecker's canonical form and the $Q Z$ algorithm. Linear Algebra Appl., 28:285-303, 1979. 